\title{
A Cluster based Routing Technique to Enhance the Lifetime of Wireless Sensor Networks
}

\author{
Deepa V. Jose \\ Christ University \\ Hosur Road, Bangalore, India
}

\begin{abstract}
The latest century experiences extensive amenities aided by Wireless Sensor Networks applications that make our lives more comfortable and secure. The small size and low cost of the sensor nodes added to its ease of deployment where human intervention is quite risky or even impossible. Wireless Sensor Networks are used in a wide variety of applications where continuous sensing of data is required hence retaining the energy of the network is very important to prolong the network lifetime. But its major drawback is the resource constraint nature; as the batteries which are the power sources, have very low capacity, it is important to ensure efficient energy consumption so as to enhance the lifetime of the entire network. Adopting clustering methods reduces energy consumption of individual nodes and can maximize the network life time. This paper proposes a clustering algorithm by incorporating unequal clustering technique in Hybrid Energy Efficient Clustering which reduces the hotspots and improves the lifetime of the Wireless Sensor Network.
\end{abstract}

\section{General Terms}

Clustering, Unequal clustering, Energy efficiency, Wireless sensor networks.

\section{Keywords}

Energy efficiency, Sensor nodes, Lifetime, Wireless sensor networks, Energy consumption, Sink nodes, Base station, HEED, Applications of WSNs, Classification of clustering algorithms

\section{INTRODUCTION}

Wireless Sensor Networks consists of a large number of small sensor nodes deployed in an area called the sensor field. These sensor nodes will be able to sense or monitor the environment by measuring the different physical parameters like temperature, humidity etc as required by the specific application for which it is used. The sensed data will be send to the sink nodes which is connected to the base station and to the communication medium from which the information can be accessed by the different personal digital assistants[1].Figure 1 depicts a typical WSN scenario. Being resource constraint, it's always inevitable to have less energy consumption for communication in order to prolong the network lifetime. The architecture of sensor node consists of four major units as represented in Figure 2.Each unit has its own role in reducing the network's energy consumption.

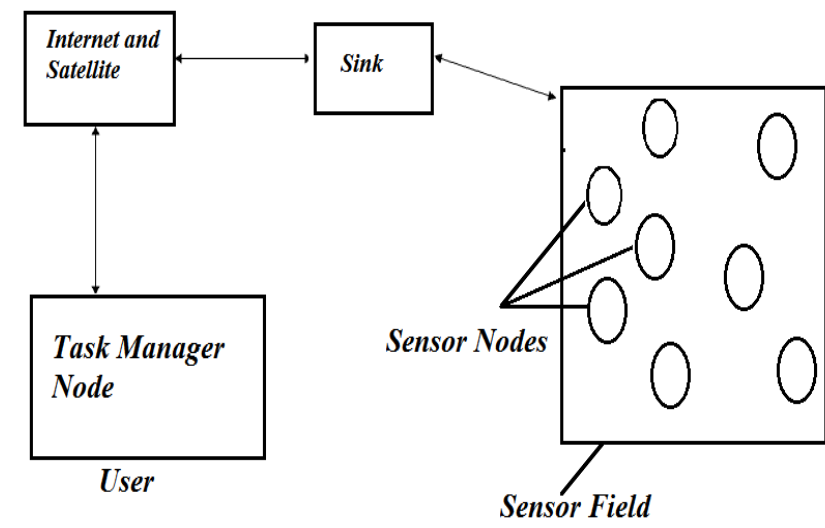

Fig 1: Wireless Sensor Networks Scenario

Immense applications in today's world are aided by WSNs like home automation, patient monitoring, and surveillance applications etc. An overview of the various WSN application areas is given in Figure 3.

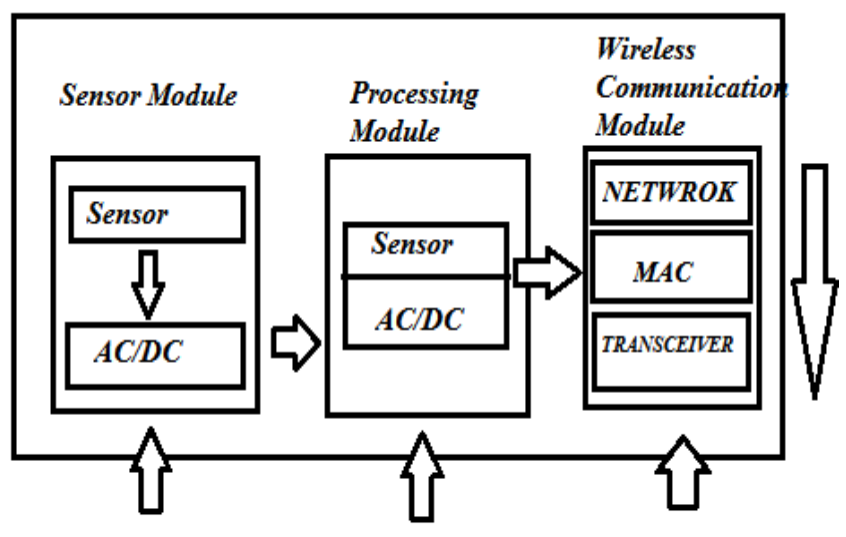

Power Provision Module (battery Supply)

Fig 2: Wireless Sensor Node Architecture 


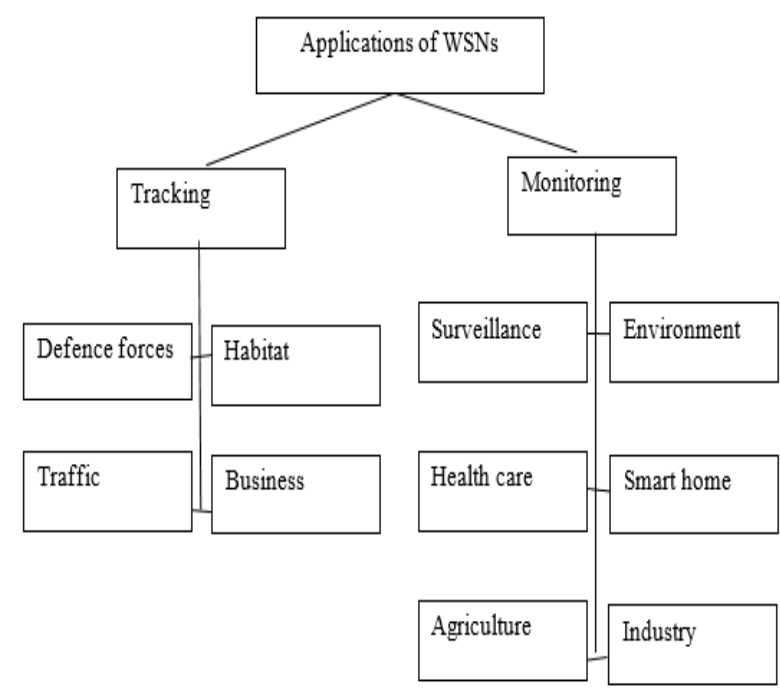

Fig 3: Applications of WSNs

Clustering is a commonly used and proven energy efficient mechanism in WSNs. In order to maximize the energy efficiency of the sensor nodes, they can be hierarchically grouped into clusters[2]. The cluster head aggregate information from its cluster members and communicate it to the base station or sink node. A general scenario of clustering is depicted in Figure 3.

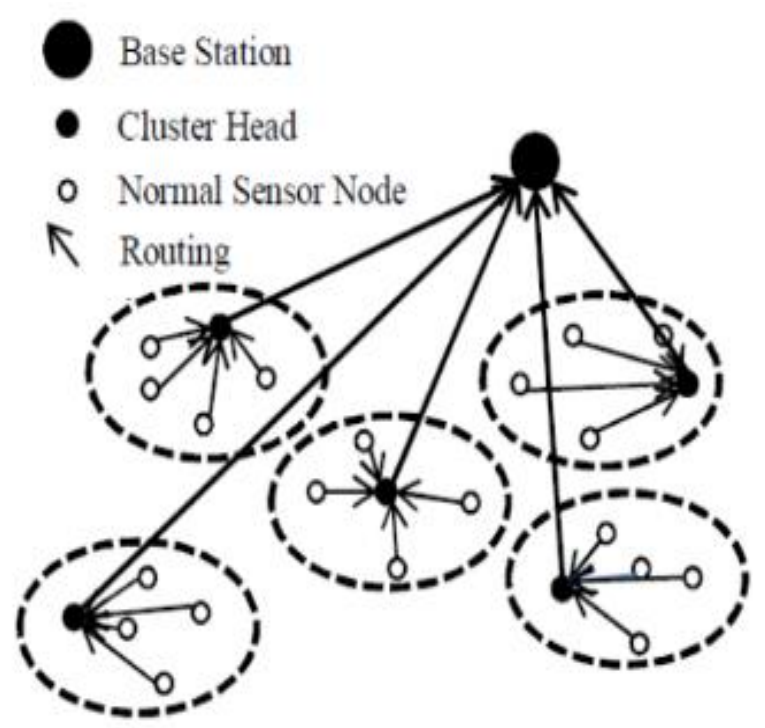

Fig 3: Clustering in WSNs

This reduces the need of all the sensor nodes participating in data transmission and thereby reduces the energy wastage. The clustering technique vary in their mode of clustering, cluster head selection criteria, optimal number of cluster heads and cluster members.

There are different types of clustering like equal clustering and unequal clustering[3]. Majority of the clustering techniques adopts the equal clustering strategy so as to have uniform number of cluster members in each cluster. But his ignores the position of the base station and the issues related to the hotspots which can ultimately terminate the network connectivity. Such problems can be avoided using unequal clustering where in the base station location is considered while selecting the cluster heads. In this method the number of cluster members also differs in each cluster so as to reduce the overload of cluster heads near to the base station. A classification of the clustering algorithms proposed by Kazerooni[4] is given in Figure 4.

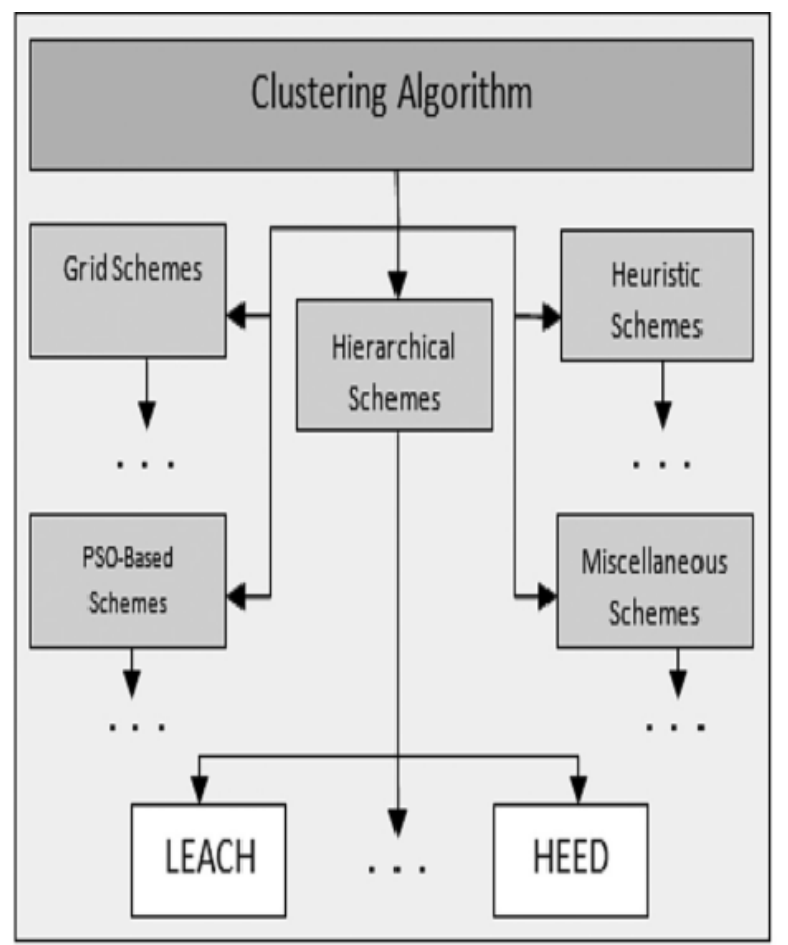

Fig 4: Classification of Clustering Algorithms

The organization of this paper is as follows. Section 2 gives a gist of the various related works in this field. The aim of this research to propose an energy efficient clustering technique so as to increase the lifespan of the WSNs. Section 3 mentions the proposed work followed by the Performance Analysis in Section 4 and Conclusions in Section 5.

\section{RELATED WORK}

LEACH[5] is a traditional cluster based routing protocol but its cluster head selection is based on a probability function which can lead to the selection of nodes with less energy also as cluster head. LEACH used equal number of clusters in order to have load balance among cluster heads; but did not take into consideration the overburdening of cluster heads near base station which can terminate the network lifetime easier. HEED[6], another energy efficient clustering protocol considers a combination of two factors like residual energy of the node and distance to the base station etc for cluster head selection which gave better network life span.

Vyas and Chouhan [7] did a survey on the clustering techniques used in WSNs for the maximum efficient energy utilization of the sensor nodes. According to them the two major classifications of clustering are centralized and distributed which is further subdivided into probabilistic and non probabilistic categories based on the criteria for cluster head selection. A comparative analysis of the clustering algorithms based on various parameters like QoS, time complexity, cluster topology, cluster head rotation etc is also carried out. Vinay et al. [8] presented a taxonomy of the clustering algorithms and the timeline of LEACH and its descendants. A survey of the clustering routing protocols in WSNs was carried out by Liu[9].The author presented a taxonomy of the clustering methods in WSNs and the classification of different cluster based routing protocols in 
WSNs. Selvi and Manoharan[2] discussed about the issues of equal clustering. The authors gave insight into the benefits of unequal clustering and presented comparative analysis of unequal clustering algorithms EEUC, MRPUC, EEDUC, EDUC, UHEED, UCR and UCS. A comparison of HEED based protocols is given in [10] where the authors proposed a variation of RHEED called ERHEED.

For enhanced lifetime and security in WSNs lifetime Jain et al.[11] proposed an query driven distributed clustering approach. According to this approach cluster heads are selected periodically. A rotated cluster head selection is tried out by Aierken et al. [12] which improved the lifetime by reducing hot spot issues compared to HEED protocol. According to the authors this approach consists of selection of cluster head, formation of clusters and rotation of the cluster head in case the current cluster head's energy go below a threshold value. Ever et al. [13] proposed a method to calculate the right cluster size from the base station. This unequal clustering eventually solved the hotspot issues to a great extend. Another unequal clustering and routing to solve the hotspot issue was carried out by Dhanpal et al.[14]. This method was experimented for non uniform distribution of nodes. According to the authors the proposed algorithm balances the energy consumption among the cluster heads effectively.

\section{PROPOSED ALGORITHM}

The proposed algorithm is an enhancement of HEED protocol proposed by Younis and Fahmy[6] which use an architecture where distributed clustering is performed for a precise number of iterations. One of the main goals of HEED is to form clusters in such a way that among the $n$ sensor nodes deployed every node is able to communicate with its cluster head in single hop and each belongs to exactly one cluster. A probabilistic method considering the residual energy of the sensor node and its proximity to the base station is used for cluster head selection. Some of the assumptions made for the sensor network are mentioned below.

- All the sensor nodes are quasi-stationary and they have similar capabilities.

- Symmetric links are used for communication.

- All nodes are location unaware.

- The batteries are non re-chargeable and are left unattended after deployment.

- The number of transmission power levels is fixed for each node.

The procedure of HEED protocol consists of three main phases; an Initialization phase where sensor nodes probabilities to become cluster head is calculate, Main processing phase and the Finalization where the clustering is performed. According to HEED, if TCP is the duration for clustering and $T_{N O}$ is time interval for network operation, the clustering and cluster head selection is invoked every $T_{C P}$ $+T_{N O}$ seconds. The clustering needs $N_{i t e r}$ iterations with a time gap $t_{c}$. Initially the cluster heads probability $C_{p r o b}$ is set to be $5 \%$ of $n$ and in later stages $\mathrm{CH}_{\text {prob }}$, is calculated as follows;

$$
C H_{\text {prob }}=C H_{\text {prob }} \times \frac{E_{\text {resi }}}{E_{m}}
$$

A predefined value $C_{p r o b}$ is the initial probability, $E_{\text {resi }}$ is the residual energy and $E_{m}$ is the maximum energy of the $n$ sensor nodes. During the main processing phase cluster head selection takes place and in the finalization stage all cluster members will join one cluster and in case any one is left out because of coverage issues, they will declare themselves as cluster heads and participates in communication. Even though
HEED makes sure that overlapping of nodes doesn't happen, it doesn't handle the hotspot problem which is a major threat for network lifetime termination.

In order to solve this issue an approach is proposed in this paper wherein unequal clustering is introduced. This will reduce the load on cluster heads near base station ensuring better load balance which ultimately increase network lifespan. Initial settings are kept similar to that of HEED including the network energy model. Sensor nodes are deployed in a random and uniform manner in an $\mathrm{A} \times \mathrm{A} \mathrm{m} \mathrm{m}^{2}$ which are supposed to be stationary. Base station is located at the coordinate position $(20,80)$ of the deployed region. The radio energy dissipation model assumes error free communication links and uses both free space and multipath channel model mentioned by Younis and Fahmy[6]. In order to run the transmitter and the receiver circuitry, the electronic energy spent by the sensor node $E_{\text {elec }}=50 \mathrm{~nJ} / \mathrm{bit}$. $\mathrm{E}_{\mathrm{a}}$, the energy spent by the transmitter amplifier is dependent on the distance' $d$ ' between the sender and the receiver. i.e. $E_{a}=E_{f} s$ for a free space model when $\mathrm{d}<\mathrm{d}_{0}$ and $\mathrm{E}_{\mathrm{a}}=\mathrm{E}_{\mathrm{mf}}$ for $\mathrm{a}$ multipath model when $\mathrm{d} \geq \mathrm{d}_{0}$, where $\mathrm{d}_{0}=75 \mathrm{~m}$ is a constant distance. $\mathrm{E}_{\mathrm{f} \mathrm{s}}=10 \mathrm{pJ} / \mathrm{bit} / \mathrm{m}^{2}$ and $\mathrm{E}_{\mathrm{mf}}=0.0013 \mathrm{pJ} / \mathrm{bit} / \mathrm{m}^{4}$. Based on the above assumptions for the radio model, for transmitting a k-size packet over a distance $d$, the amount of energy consumed for transmission $\mathrm{E}_{\mathrm{Tx}}$, can be calculated as:

$E_{T X}=\left(E_{\text {elec }} \times k\right)+\left(E_{a} \times k \times d^{n}\right)$

where, $\mathrm{n}$ is assigned 2 for the free space model and 4 for the multipath model. The energy spent to receive a k-bit size message $\mathrm{E}_{\mathrm{Rx}}$ is calculated as:

$E_{R X}=E_{\text {elec }} \times k$

The proposed algorithm incorporates a waiting time for reclustering to avoid two nodes sending the message at the same time and a cluster radius to ensure unequal cluster size to reduce the size of clusters near base station. The initialization phase starts with the base station broadcasting a signal. All nodes calculate their distance to the base station based on the received signal strength. Within its communication range ' $r$ ', each node transmits a message consisting of its id and the residual energy. A waiting time $t_{i}$ is also set for broadcasting the cluster election to avoid multiple nodes sending messages at the same instance. In order to have this, a random value between $[0.9,1]$ is assigned to $\mathrm{V}_{\mathrm{r}}$. The waiting time[3] is calculated as;

$t_{i}= \begin{cases}\frac{E_{i a}}{E_{i r}} T_{2} V_{r}, & E_{i r} \geq E_{i a} \\ T_{2} V_{r}, & E_{i r}<E_{i a}\end{cases}$

Where $\mathrm{E}_{\mathrm{ia}}$ is the average residual energy, $\mathrm{E}_{\mathrm{ir}}$ is the residual energy of node $i, T_{2}$ is the time duration, the random value between $[0.9,1]$.Initial steps of the proposed enhanced HEED is similar to HEED where the process happens in iterations. The average minimum rechargability power (AMRP) is calculated. Every round will be initiated with the clustering mechanism leading to clustering and later to data transmission through intra-cluster and inter-cluster mechanisms. After the initial round, a random period of waiting time is introduced as in equation (4) for re-clustering and a competition radius as in equation (5) is used to have non uniform cluster sizes[14].

$R_{C i}=\left[1-\propto \frac{d_{\max }-d_{i}}{d_{\max }-d_{\min }}\right] \mathrm{R}_{\mathrm{m}}$

where $d_{m a}$ and $d_{m i}$ are the maximum and minimum distance of the sensors from the base station, $d_{i}$ is the distance of the sensor nodes to the base station, $\alpha$ is a random value which 
belongs to $[0,1], R_{m}$ is the maximum competition radius allowed. For the simulation $R_{m}$ is taken as the diagonal distance divided by ten.

The aim of this research work is to avoid the premature death of the cluster heads near the base station by reducing the overload in them. Using the above mentioned cluster radius as in equation (5), the clusters near the base station will be small and obviously the cluster heads have to manage less number of nodes in the cluster saving the energy for forwarding the data.

The main difference between HEED and the proposed algorithm is the waiting time and unequal cluster size clustering. The proposed algorithm creates clusters of unequal sizes. This approach also makes sure that the clusters nearer to the base station have lesser members in it which eliminates the untimely death of the nodes close to the base station Hence the overburden of such clusters will get reduced and better load balance can be achieved which will increase the efficiency of the WSNs.

The summary of the proposed algorithm is given below.

Step1: Initially all sensor nodes calculates their proximity to the base station and their energy levels and does clustering according to HEED

Step 2: In the next round re-clustering is done based on the competition radius given in equation (6) after waiting for time $t_{i}$ as in equation (5).

Step 3: Intra cluster routing is performed and the cluster head transmits the information to the nearest cluster head.

Step 4: If the CHs energy level goes beyond a predefined threshold hold value, the cluster member with the next highest energy level and shortest distance to the base station will continue to be the cluster head for that round. After that, go to step 2 for next round.

Step 5: After $\mathrm{CH}$ selection and clustering is done, data is routed (intra-cluster and inter-cluster) to the base station.

\section{PERFORMANCE ANALYSIS}

The proposed algorithm is compared with HEED based on parameters total energy consumed and the number of live nodes. The simulation parameters are set similar to that of HEED. The simulation parameters are given in Table 1.

Table 1. Simulation parameters

\begin{tabular}{|l|l|}
\hline Parameters & Values \\
\hline Area of simulation & $100 \mathrm{~m} \times 100 \mathrm{~m}$ \\
\hline Base station location & $(20,80)$ \\
\hline Number of nodes & 50 \\
\hline $\mathrm{E}_{\text {elec }}$ & $50 \mathrm{~nJ} / \mathrm{bit}$ \\
\hline $\mathrm{E}_{\mathrm{fs}}$ & $10 \mathrm{pJ} / \mathrm{bit} / \mathrm{m}^{2}$ \\
\hline $\mathrm{E}_{\mathrm{mp}}$ & $0.0013 \mathrm{pJ} / \mathrm{bit} / \mathrm{m}^{4}$ \\
\hline Initial energy & $5 \mathrm{~J}$ \\
\hline Deployment model & Random \\
\hline
\end{tabular}

The different parameters used for performance evaluation of the proposed algorithm are the lifetime of network, and average residual energy of the network. For the simulation results presented here, the lifetime of the network is calculated as the time when fifty percentages of nodes get depleted. The average energy of the nodes at the end of the simulation is taken as the average residual energy of the nodes. Figure 5 represents the random deployment of 50 sensor nodes over $100 \mathrm{~m} \times 100 \mathrm{~m}$

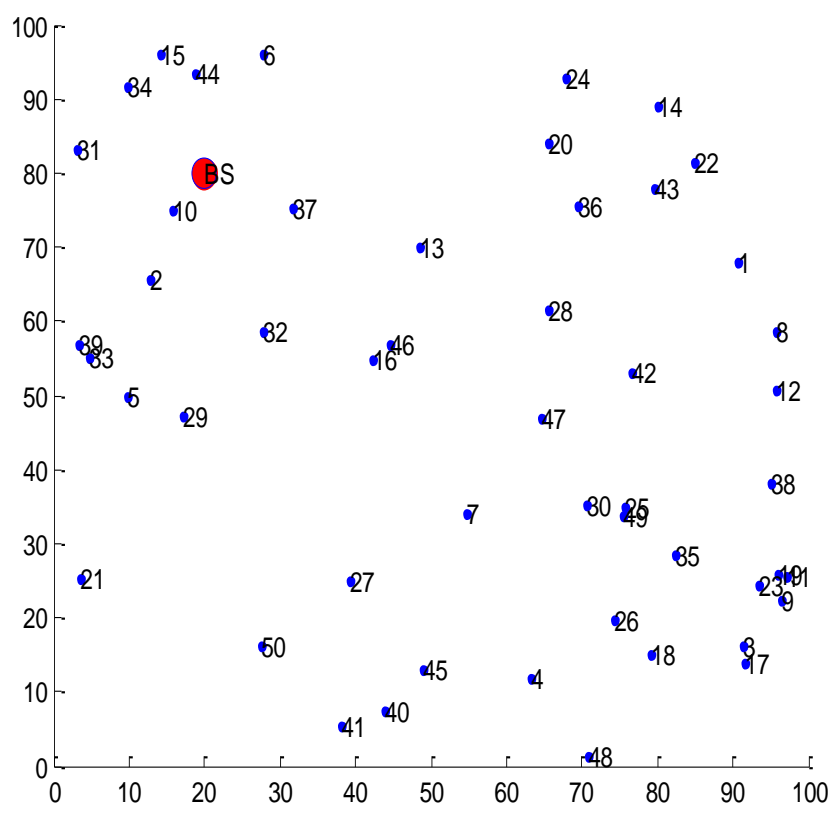

Fig 5: Random deployment of 50 sensor nodes in an area $100 \mathrm{~m} \times 100 \mathrm{~m}$.

Figure 6 represents the comparison of the number of live nodes for the HEED and the proposed algorithm at different rounds and Figure 7 represents the comparison of the residual energy of the nodes for the same.

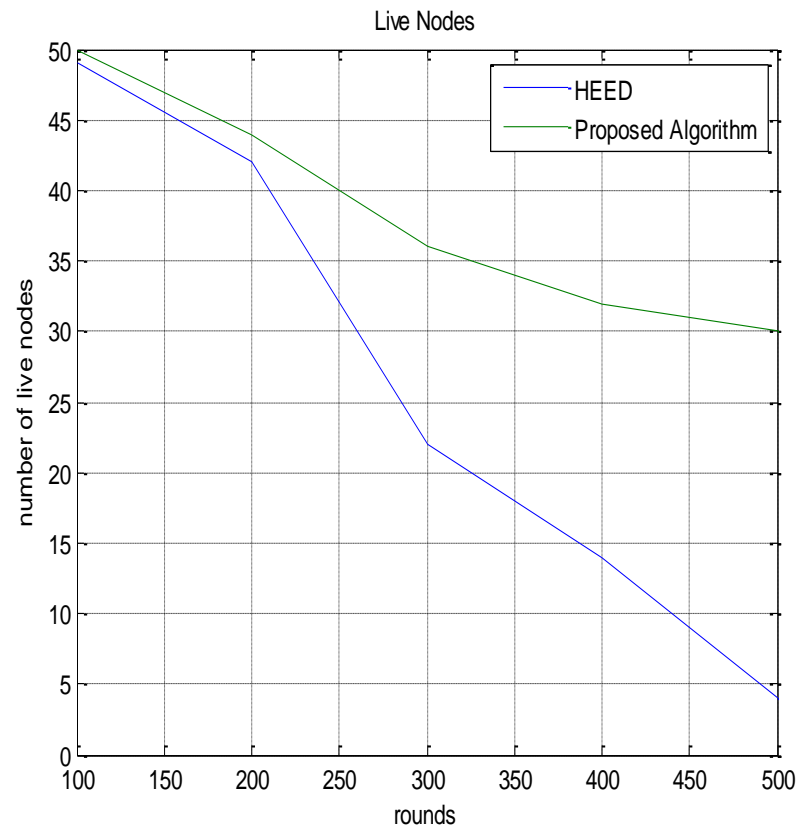

Fig 6: Number of alive nodes versus rounds $(\mathrm{N}=50$ and $A=100 \mathrm{~m} \times 100 \mathrm{~m}$ ) 


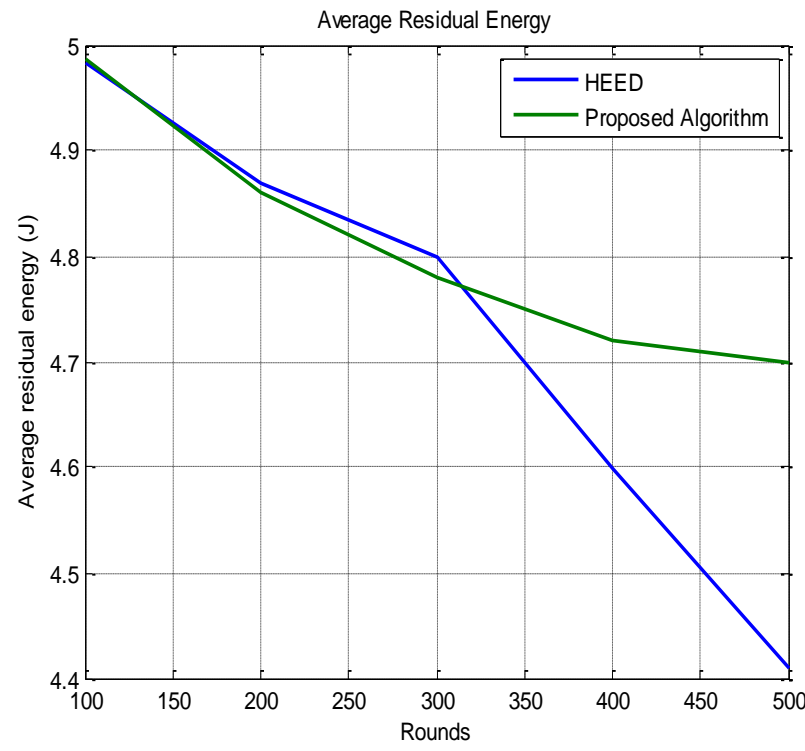

Fig 7: Average energy of the nodes versus rounds $(\mathrm{N}=50$ and $A=100 \mathrm{~m} \times 100 \mathrm{~m}$ )

Based on Figure 6 and 7 it is evident that the number of live nodes and the residual energy of the nodes in each round is more in the case of proposed algorithm. The simulation results proves that the unequal clustering have achieved better load balance among cluster heads resulting in reducing the hotspots and hence increased lifespan.

\section{CONCLUSIONS}

This paper proposes an unequal clustering algorithm to reduce the size of the clusters near the base station. Usually the cluster heads near the base station will be constantly busy with maintaining its cluster members as well as participating in relay of data from other clusters to the base station. Because of this reason they used get depleted earlier resulting in the premature termination of the network lifetime. The proposed approach reduces the encumbrance of the cluster head near base station region which eventually reduced the hotspot issues and resulted in better life time.

\section{ACKNOWLEDGEMENT}

This project is supported by Faculty Research Program under Minor Research Projects Scheme of Christ University, Bengaluru.

\section{REFERENCES}

[1] N. a Pantazis and D. D. Vergados, "A survey on power control issues in wireless sensor networks," IEEE Commun. Surv. Tutorials, vol. 9, no. 4, pp. 376-380, 2010.
[2] G. V. Selvi and R. Manoharan, "A Survey of Energy Efficient Unequal Clustering Algorithms for WSNs," vol. 79, no. 1, pp. 1-4, 2013.

[3] D. V Jose, "An Improved Hybrid Energy Efficient Clustering Technique to Enhance the Lifespan of Wireless Sensor Networks," vol. 135, no. 10, pp. 43-49, 2016.

[4] A. Kazerooni, H. Jelodar, and J. Aramideh, "Leach and Heed Clustering Algorithms in Wireless Sensor Networks: a Qualitative Study," Adv. Sci. Technol. Res. J., vol. 9, no. 25, pp. 7-11, 2015.

[5] W. B. Heinzelman, a. P. Chandrakasan, and H. Balakrishnan, "An application-specific protocol architecture for wireless microsensor networks," IEEE Trans. Wirel. Commun., vol. 1, no. 4, pp. 660-670, 2002.

[6] O. Younis and S. Fahmy, "HEED: A Hybrid, EnergyEfficient, Distributed Clustering Approach for Ad-hoc Sensor Networks," vol. 238294, pp. 1-36.

[7] P. Vyas and M. Chouhan, "Survey on Clustering Techniques in Wireless Sensor Network," vol. 5, no. 5, pp. 6614-6619, 2014.

[8] V. Kumar, S. Jain, S. Tiwari, and I. Member, "Energy Efficient Clustering Algorithms in Wireless Sensor Networks : A Survey," vol. 8, no. 5, pp. 259-268, 2011.

[9] X. Liu, "A Survey on Clustering Routing Protocols in Wireless Sensor Networks",Sensors, 2012.

[10] Z. Ullah, L. Mostarda, R. Gagliardi, D. Cacciagrano, and F. Corradini, "A comparison of HEED based clustering algorithms - introducing ER-HEED," 2016.

[11] A. K. Jain, V. Chargotra, M. Student, and M. Mark, "Reliable Sta ate-full Hybrid Energy E fficient Distributed clust tering protocol for Wire less Sensor Networks : RS-HEED,” pp. 396-401, 2015.

[12] N. Aierken, R. Gagliardi, L. Mostarda, and Z. Ullah, "RUHEED- Rotated Unequal Clustering Algorithm For Wireless Sensor Networks."

[13] R. Luchmun, L. Mostarda, A. Navarra, and P. Shah, "UHEED - AN UNEQUAL CLUSTERING ALGORITHM FOR WIRELESS SENSOR NETWORKS,” 2010.

[14] D. K. Dhanpal, A. Joseph, and A. Panicker, "An Energy Efficient Unequal Cluster Based Routing Protocol For WSN With Non-Uniform Node Distribution," vol. 4, no. 5,2015 . 